\title{
A Multi-Layer Approach for Cross-Technology Communication in a Pervasive Community
}

\author{
Enrico Dressler, Raphael Zender, Ulrike Lucke and Djamshid Tavangarian \\ University of Rostock, Faculty of Engineering, Institute of Computer Science, \\ Chair of Computer Architecture, Rostock, Germany \\ E-mail: firstname.lastname@uni-rostock.de
}

\begin{abstract}
This paper presents a middleware and an application scenario for communication in a pervasive community which is characterized by the use of different network and service technologies. To achieve the required cross-technology communication, we organize devices and applications as members of cells with homogeneous network interfaces, and we combine these cells to a heterogeneous ensemble by a General Purpose Access Point (GPAP). The GPAP enables and organizes a flexible service-based communication between members of different cells. This paper focuses an interconnection between Bluetooth and IP-based networks using service proxying between Bluetooth SDP (Service Discovery Protocol) and Web Services. However, it can be used as a base for service proxying with a variety of other network and SOA technologies. Furthermore, we combine several ensembles by our service-oriented middleware to build a community and enable the communication between devices of different ensembles. Moreover, we look forward to explore and allow seamless roaming for mobile devices as well as their provided services inside the community. For evaluation of our middleware we use cross-technology messaging as an application scenario for a general cross-technology video and audio communication.
\end{abstract}

Keywords: pervasive community, cross-technology communication, Bluetooth, SOA, Web Services, service proxying, mobile services, roaming

\section{INTRODUCTION}

The continuing technological progress in microelectronics with decreasing dimensions of components and devices leads information processing to be integrated into every day objects and activities. The vision of small, inexpensive, and robust networking devices that provide their abilities in terms of services and support users in their everyday work in a proactive and intelligent manner will significantly change our environments, but is associated with the usage of heterogeneous network and service technologies which are not compatible at all.

Wireless communications, especially Wireless Personal Area Networks (WPANs), Wireless Local Area Networks (WLANs), and cellular networks enjoy an unprecedented growth over recent years so that millions of people can exchange information every day using mobile phones, notebooks, personal digital assistants (PDAs), and sensors in their pervasive environment. This widespread and integrated use of different wireless network technologies allows devices in our environment to use a specialized communication technology for a certain task.

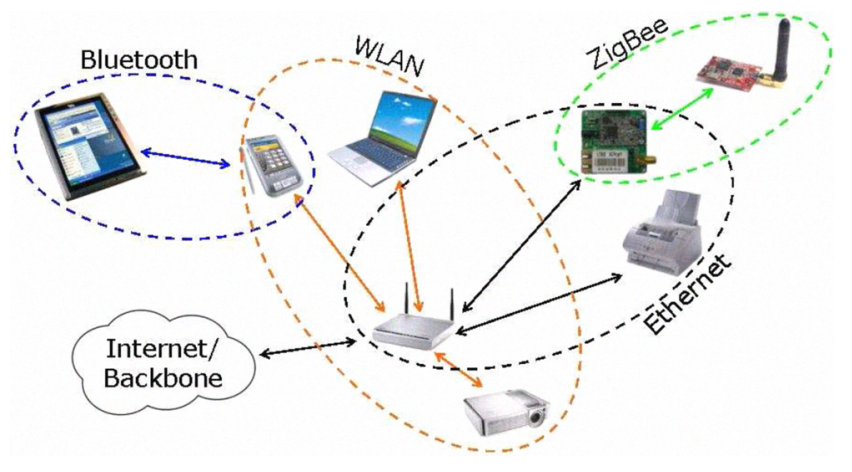

Fig. 1. Today's cell based communication inside an ensemble emerged by a pervasive environment.

A typical infrastructure of a pervasive environment, as depicted in figure 1, is arranged on the basis of an IP router connected to the Internet by wire. Further devices using wired Ethernet (like a printer or an embedded device) and devices using WLAN (like a notebook or a PDA) can be directly integrated into the network. Communication between mobile devices using Bluetooth and ambient sensors using ZigBee is possible as well. Thus, different cells evolve, characterized as locally closed collections of devices interconnected by the same wired or wireless network and service technology. Thereby, wireless cells are typically dynamic because devices spontaneously enter or leave the cell's scope. Wired cells may be dynamic as well, because they can be powered on/off or cables can unpredictably be plugged or unplugged. A communication between members of directly overlapping cells is partially possible today, but not a communication between members of separated cells. For instance, a communication between a ZigBee and a Bluetooth device in figure 1 is not possible so that communication between any devices on network and service layer in a pervasive environment is still missing today.

To enable such a communication a combination of different homogeneous cells is necessary to bridge cells depending on their network technologies in a centralized or decentralized way. For example, we use a centralized General Purpose Access Point (GPAP) [5] and service proxying for Bluetooth SDP and Web Services to combine Bluetooth and IP-based cells for our cross-technology communication scenario. Thereby, a heterogeneous ensemble evolves out of homogeneous cells. 
Furthermore, service proxying can be used to combine multiple separated ensembles to a community [6] that allows not only data exchange between any devices inside an ensemble but also communication far beyond the physical borders of local ensembles. The realization of such a communication is the base for our cross-technology communication scenario.

At the beginning, this paper presents an application scenario for cross-technology communication in a pervasive community. Afterwards, related work and current limitations in the area of heterogeneous network and service technologies, especially inter-working of WLAN and WPAN technologies, will be described. Thereafter, the paper introduces our prototypical network/service middleware that expands related approaches. The paper ends with a conclusion and future work.

\section{Cross-Technology Communication Scenario}

The possibilities of available mobile phones, notebooks, and personal digital assistants to support the user in his every day work and mobile information retrieval lead to the desire of using these devices for communication with other users inside an ensemble as well as inside a community. Such a user-to-user communication can be build-up on a text, video or audio chat using different end-user devices. For instance, inside a university chats can be used to exchange messages and data between learners and teachers. Inside a lab, a Bluetooth cell allows direct communication between devices using the Bluetooth network technology and SDP services. On a corridor, a WLAN cell can be build-up which typically uses Web Services as service technology. These cells can be combined to an ensemble by a GPAP using service proxying to allow a transparent communication between devices of both cells. Thus, a transparent communication between devices using SDP and Web Services in a small-sized area can be realized. The combination of multiple areas (ensembles) of the campus leads to a community which allows communication not only between different network and service technologies but also between separated physical sites of the campus as well as virtual areas like 3D worlds (e.g. Second Life) to enable lessons with remote participants. By using our approach to combine homogeneous cells to a heterogeneous ensemble and to combine ensembles to a community, it doesn't make a functional difference to the user whether he communicates with another local or distant user, just the latency will be increased insignificant.

In this scenario some of the end-user devices use IPbased technologies like WLAN or Ethernet. Other devices use network technologies like Bluetooth or ZigBee, whose communication is typically not based on IP-addresses. This interoperability of different network technologies simultaneously leads to the use of different service technologies. Thus, for a chat between the PDA and the notebook inside ensemble 1, displayed in figure 2, a middleware is needed that bridges between WLAN and Bluetooth network technologies and simultaneously between Web Services and Bluetooth SDP services. For communication between devices of remote ensembles, for example the PDA in ensemble 1 and the notebook

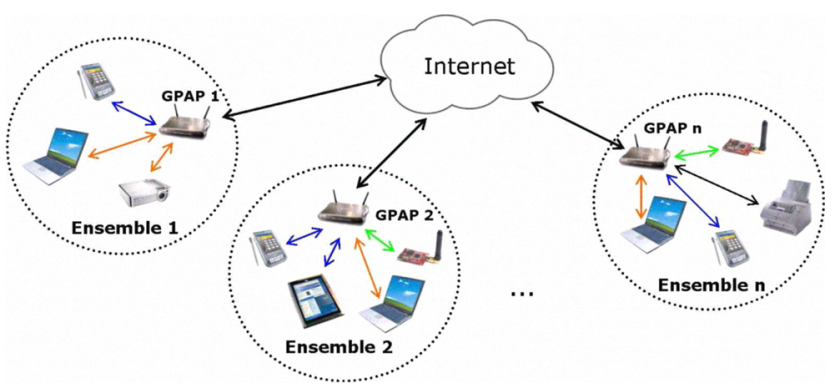

Fig. 2. Cross-technology communication inside an ensemble and expanded to multiple ensembles inside a community.

in ensemble 2, the middleware needs a functionality to link remote ensembles to a community. Further functionality will be needed for expansion of text chat to video and audio chat, for instance to submit a stream of a lecture from a teacher to learners. Thereby, real-time, mobility, routing, and handoff requirements have to be considered, while allowing a user to transparently move from ensemble to ensemble inside a community during communication.

\section{RELATED WORK}

A key feature of our cross-technology communication scenario is the inter-working of various WPAN and WLAN technologies in a pervasive community. Given the importance within the WPAN operating space, availability of devices, and intensive research activities, the industry standard Bluetooth is the best example for a WPAN technology. Bluetooth is a robust and flexible low cost, low power, and short-range radio communication standard defined by the Bluetooth Special Interest Group (SIG) [8]. It can be found in many consumer electronics such as cell phones, PDAs, and notebooks and enables an easy and direct communication between them. Independence of infrastructure and required IP-addresses as precondition for communication is the main goal of the Bluetooth technology. But the short range of direct Bluetooth communication is a limiting factor for Bluetooth applications and makes a combination with other technologies necessary. In heterogeneous networks, WLAN and WPAN technologies differ in terms of power consumption, coverage range, data rate, and costs, but they can be used to complement one another by different approaches to realize a communication between Bluetooth and WLAN enabled devices and between remote Bluetooth devices using a WLAN backbone.

A general approach for integration of Bluetooth devices into IP based networks is an all-over-IP issue. For this approach the Bluetooth SIG has published a native way for carrying IP traffic over Bluetooth by a protocol called Bluetooth Network Encapsulation Protocol (BNEP) [9], wherein unmodified IP packets are encapsulated in Bluetooth packets which are carried over Bluetooth links. This ability to communicate with a WLAN allows WPANs to take advantage of services such as printing, Internet access, and file sharing. Thereby, a network access point with a Bluetooth and a WLAN network interface is used to mediate between these network technologies. The 


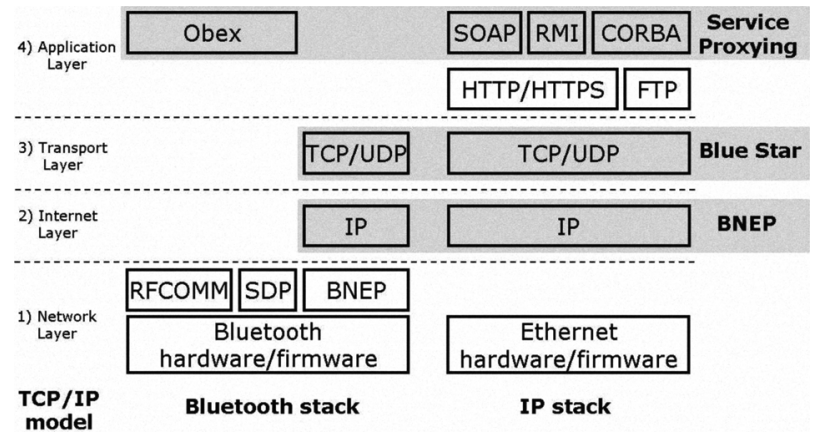

Fig. 3. Approaches for inter-working of LANs and WPANs.

connection procedure and setup time is extremely low and is therefore a very attractive option, considering the high data rates of 11-55 Mbps in traditional WLANs. This approach allows a communication between WLAN and Bluetooth enabled devices on the internet layer of the TCP/IP model and above, displayed in figure 3. But, this approach leads to different drawbacks because the use of BNEP assumes an IP stack on every device. Thus, IP, gateway and nameserver addresses must be made available on all Bluetooth enabled devices. Furthermore, the IP stack leads to the use of IP-based service technologies on Bluetooth devices so that adaptations of existing applications are now necessary for a service-based communication between Bluetooth devices. This assumption leads to software, protocol, and management overhead, typically unnecessary for common Bluetooth communication.

Current developments like BlueStar [3][4] enable such an all-over-IP approach of Bluetooth WPANs and IEEE 802.11 WLANs. They use a few Bluetooth Wireless Gateways (BWGs), whick are also IEEE 802.11 enabled so that they can serve as access point to the IEEE 802.11 wireless network. BlueStar enables low-cost and short-range Bluetooth devices to access the global Internet infrastructure by utilizing WLANbased high-powered transmitters. The BlueStar architecture is an intuitive and practical solution for IP-based communication between Bluetooth and WLAN enabled devices but doesn't allow a transparent communication between them.

The second communication approach combines Bluetooth and IP-based networks on application layer using Service proxying, displayed in figure 3 . The operation mode of this approach is displayed in figure 4 [7]. It uses an essential method of locating services in mobile Bluetooth ad hoc networks and utilizes proxies to expand the service based communication, to discover remote devices, and to make a setup of communication links between them possible. Therefore, each proxy has to search for available Bluetooth SDP services of local devices and to offer them to the other proxy. Thus, a service SDP_A provided by the TabletPC within the range of Proxy_1 can be forwarded to Proxy_2. Then Proxy_2 provides this service to a local PDA. Finally, the PDA can search for SDP services and use the remote service SDP_A. In the other direction, service SDP_B must be made available for the TabletPC on Proxy_1. For service usage, a communication

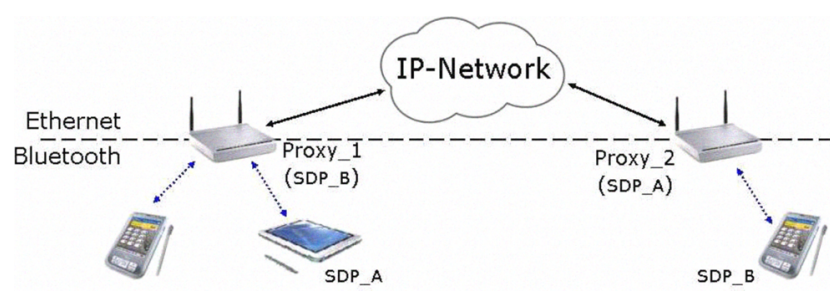

Fig. 4. Service proxying for Bluetooth SDP.

channel would then be set up between the TabletPC and the remote PDA by the proxies that transparently by-pass the communication over the IP network. Thus, applications on either end will communicate seamlessly and transparently with one another, unaware that the devices are not within radio range. So the communication range of Bluetooth devices can be increased by a large-scale IP-based backbone network. However, a seamless and transparent communication between Bluetooth SDP and IP-based Web Services is still missing.

A service proxying approach for combining Bluetooth SDP and IP-based Web Services, implemented with our middleware and explained in detail in the next chapter, could be adopted as candidate for combination of a variety of incompatible SOA technologies. Many of them have been established for dedicated application areas, like Open Services Gateway Initiative (OSGI) [12] for car and home automation, Jini [13] and Universal Plug and Play (UPnP) [14] for device use in IP-based networks, Bonjour [15] in particular for print services, and Web Services [16] especially for the business domain. Many existing approaches for interoperability of SOA technologies use bridge nodes to map services of technology A to services of technology B. Bridges act as provider and consumer in the $\mathrm{A}$ and $\mathrm{B}$ networks and allow the usage of services from network A in service network B and vice versa. But, full interoperability between $t$ available SOA technologies requires $\left(\begin{array}{l}t \\ 2\end{array}\right)$ bridge nodes. An advanced approach [6] uses a surrogate approach and distinguishes between real and virtual services to significantly reduce the number of needed service bridges. This approach will be used in the following section to realize our cross-technology communication scenario by means of our middleware. Without this service-proxying approach, a service consumer still needs to use the same technology as the service provider and has to adapt to multiple SOA technologies in a pervasive environment depending on the application area. Thus, the advantage of the abstraction from devices would no longer be given by using services.

\section{SERVICE ENHANCEMENT OF THE GPAP MIDDLEWARE}

To realize our cross-technology communication scenario by using service-proxying between Bluetooth SDP and Web Services a middleware for combining network and service technologies is required. The middleware is based on a network and a service layer, running on the proxies. End-user devices remain unmodified in relation to the middleware. Thus, regular mobile devices, equipped with Bluetooth interfaces can use available SDP services to transparently obtain a service of 


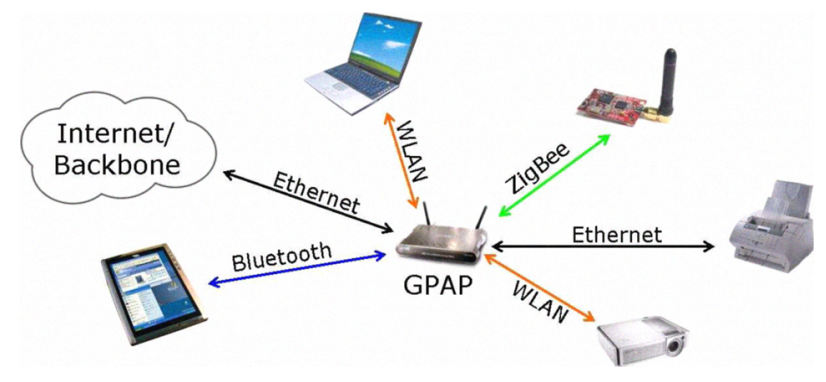

Fig. 5. A General Purpose Access Point (GPAP) using the middleware to combine homogeneous cells to a heterogeneous ensemble.

another service technology while the proxies enable bridging between both service technologies.

\section{A. Network Layer}

The network layer of our middleware is based on the centralized GPAP approach [5]. The GPAP, displayed in figure 5 , is made up by a device with numerous network interfaces (e.g. Ethernet, WLAN, ZigBee, Bluetooth), each representing a member of another homogeneous cell. By combining these homogeneous cells to a heterogeneous ensemble the notebook can send video data to the projector to make a presentation by using WLAN, share data with the TabletPC by using a combination of WLAN and Bluetooth, and obtain sensor data by using a combination of WLAN and ZigBee. Thus, a communication between all devices inside an ensemble can be realized while using different network technologies.

The network layer, using an enhanced version of the proposed Heterogeneous Context Based Routing (HCBR) architecture [5], permits the integration of all kinds of wired and wireless technologies. Additionally, the layer organizes homogeneous cells to a heterogeneous ensemble by bridging the communication between different cells and allows devices of different cells to communicate with each other. The central point of this layer is the HCBR core which supports a modular enhancement by a plugin concept to support new network technologies and functionalities. This core can be extended by two types of plugins: device plugins and HCBR plugins, and is also responsible for their collaboration.

For every new technology a device plugin is necessary to encapsulate special characteristics for arranging the communication with a specific network interface. Characteristics in data transport are treated as well. Thus, device plugins provide an intermediate layer to abstract from available wired and wireless network interfaces in the operating system.

HCBR plugins are used to enhance the HCBR core with new functionalities. The most important functionality is the realization of bridges between different homogeneous cells. To reduce the amount of necessary bridges, we use Ethernet as a basic technology and accordingly implement bridges between Ethernet and other technologies only. Thereby, we need bridges such as Bluetooth-Ethernet, ZigBee-Ethernet, but do not need bridges like Bluetooth-ZigBee implicitly (only for hard performance requirements), because this functionality can be composed by a Bluetooth-Ethernet and a ZigBee-Ethernet bridge as well.

Furthermore, plugins that gather and exchange information on neighbors, links, and network topology have been realized. This information is provided to other HCBR plugins, for example to a plugin which is responsible for routing in a heterogeneous network. This plugin can use the information to construct a smart metric for a routing table that uses the available network capacity of all sorts of channels inside the ensemble to significantly improve the overall capacity of the network. Hence, measure based [1] and status based methods [2] could be used to realize multi-path routing. Furthermore, we implemented an info plugin that passes information from the HCBR core to a graphical user interface in order to allow analysis of network behavior.

Another plugin for collaboration between the network and the service layer is realized that allows the service layer to use context information from the network layer and vice versa. Thereby, the network layer provides information of available routes in the network (latency, available bandwidth,...) that can be used on service layer to enrich service information with dynamic QoS parameters. In the other direction, service information can be used to optimize network routes for services with real-time requirements, for example in a future heterogeneous video or audio chat.

\section{B. Service Layer}

The service layer of our middleware is based on the Service Technology Independent Architecture (STIA) approach [6]. This layer is responsible for mapping between different service technologies, for example between Bluetooth SDP services and IP-based Web Services used in our cross-technology communication scenario. The service layer uses a Service Technology-independent Language (STiL) for translation between abstract and concrete services, and consists of three parts: the STiL Service Manager (SSM), multiple Service Technology Translators (STT), and multiple Service Technology Plugins (STP). Each of the plugins is used to discover and publish services of one specific service technology, like Jini or Web Services. Each STT is used to translate services, discovered by the service plugins, into a STiL Service description (e.g. a Bluetooth service provided by a mobile device). Thereby, context information from the network layer can be used to enrich the service description. Afterwards, the SSM uses the description to translate the service into other supported service technologies, using a specific STT (e.g. prepare an equivalent Web Service description) and publishes the service using the specific STP. The service transformation runs in the other direction in the same manner. While using STiL for temporary service descriptions, the number of needed STTs can be reduced.

To use a service of a distant ensemble, an interconnection between multiple GPAPs (ensembles) is required. We consider linked ensembles as a community. Thereby, services of an ensemble are not only translated into and provided for other service technologies inside an ensemble, but also provided 


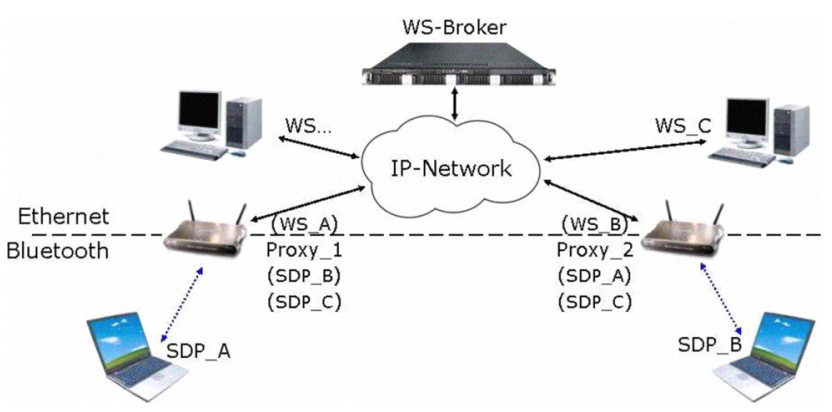

Fig. 6. Service proxying for Bluetooth SDP and Web Services.

in distant ensembles of the community. Therefore a P2Pbased exchange of STiL service descriptions between linked ensembles is used to allow an inter-ensemble service-based communication between members of different cells.

\section{EXPERIMENTAL RESULTS}

For our cross-technology communication scenario, we used the experimental set-up displayed in figure 6 with various enduser devices equipped with Bluetooth $(2,1 \mathrm{Mbit} / \mathrm{s}$ maximal data rate) or rather Ethernet (100 Mbit/s) interfaces and proxies with both interface types. To demonstrate and measure the service proxying functionality of our middleware we used a self-made Java chat application with a file-transfer function based on a Linux operating system, the BlueZ[10] Bluetooth stack and avetana[11] for BlueZ Java bindings. Two clients, a chat client for IP-based networks (using Web Services on the PCs) and a chat client for Bluetooth-based networks (using SDP services on the notebooks) have been realized.

These clients enable a direct chat and file-transfer between devices with network interfaces of the same type using the same service technology. Thus a direct chat and file-transfer between the PCs as well as nearby notebooks is possible without the proxies. To measure the bandwidth of a direct Bluetooth connection notebook A provides a service SDP_A that accepts files. Then notebook B searches for the service and uses a found service by sending a file to notebook A. Thereby, we measured a bandwidth of $154,2 \mathrm{kB} / \mathrm{s}$ of rawfile-data using a data-size greater than 10 Bytes per packet without header information and a used Bluetooth bandwidth of $158,0 \mathrm{kB} / \mathrm{s}$ with consideration of header information. The amount of the protocol overhead in this case is 2,5\%. To submit a file using Web Services PC_C provides a service that accepts files and registers the service at a Web Service broker. Then, another PC requests the service at the broker und uses it directly to submit a file to PC_C. Thereby, we measured a bandwidth of $698,5 \mathrm{kB} / \mathrm{s}$ for raw-file-data and a used bandwidth of $996,5 \mathrm{kB} / \mathrm{s}$ considering SOAP protocol overhead. This measure shows an overhead of $43 \%$ while using SOAP as communication protocol for Web Services and we noticed that using small data-chunks for data-transfers with SOAP causes an even worse use of available bandwidth.

Afterwards, we introduced the proxies to bridge between Ethernet and Bluetooth cells using service proxying between

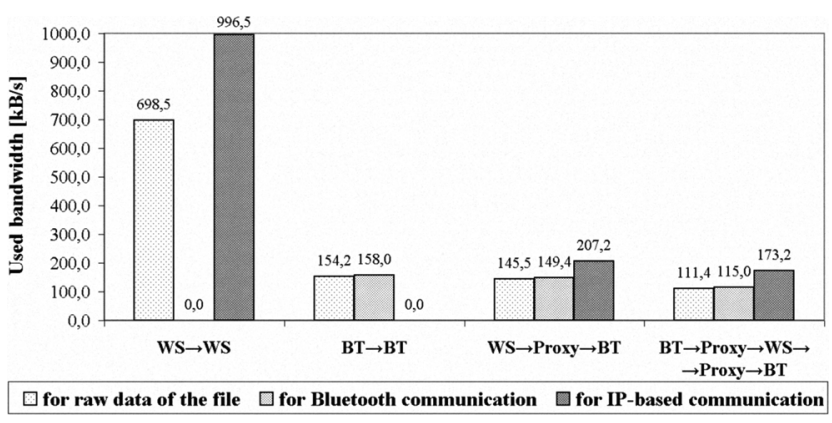

Fig. 7. Measurement results of a file-transfer using SDP and Web Services.

SDP and Web Services to transfer a file from a PC to a notebook. For this purpose, notebook A provides a local service SDP_A to accept files. Proxy_1 searches for local Bluetooth services periodically, finds service SDP_A, creates an equivalent Web Service WS_A and makes it available in the IP-based network. After searching for the service PC_C uses WS_A assistant for SDP_A and sends the data to Proxy_1 who forwards the data to the original service SDP_A. Thereby, we measured a bandwidth of $145,5 \mathrm{kB} / \mathrm{s}$ for raw-file-data.

In the last scenario we measured a file-transfer between two apart Bluetooth devices, device A and B. Therefore, service proxying between SDP and Web Services is needed twice, at Proxy_1 and Proxy_2. Once again, notebook A provides service SDP_A, Proxy_1 finds the service and makes it available as a Web Service WS_A in the IP-network. Simultaneously Proxy_2 searches for Web Services, finds WS_A and provides the service as a local Bluetooth service SDP_A which can be found and used by notebook $B$. A bandwidth of $111,4 \mathrm{kB} / \mathrm{s}$ has been measured for the raw-file-data in this scenario. Therefore, a bandwidth of $173,2 \mathrm{kB} / \mathrm{s}$ has been used for transmission in the IP network.

The measured results, as depicted in figure 7 , show that our middleware leads to efficient results in cross-technology communication between SDP and Web Services as well as proxied remote SDP services. Thereby, the necessary overhead for Bluetooth communication is very small compared to the SOAP overhead of about $55,5 \%$ in the last scenario. These results support our approach to use a specialized service technology optimized for a special network technology and link between different technologies instead of using an all-over-IP issue using Web Services on Bluetooth enabled devices.

We had to observe that service usage on both sides, SDP and Web Services, is currently not really applicable for applications with real-time requirements running on mobile devices. For example, a proxy needs more than 10 seconds to discover a new Bluetooth device and related services. So if a mobile device in range of Proxy_1 (figure 6) provides a SDP service, afterwards enters the range of Proxy_2 and leaves the range of Proxy_1, real-time requirements for video and audio communication cannot be maintained for a while.

However, our prototype shows that service proxying allows a really transparent use of local and remote services of the same service technology (SDP $\leftrightarrow \mathrm{SDP}$ ) as well as proxied ser- 
vices of different service technologies (SDP $\leftrightarrow$ WS). Furthermore, the useable bandwidth of direct and indirect communication of local as well as remote Bluetooth devices differs only marginal. The benefit of this approach and our middleware is the expandability for further service technologies to enable a pervasive community out of heterogeneous ensembles and homogeneous cells.

\section{CONCLUSION AND FUTURE WORK}

This paper presents a middleware and an application scenario for cross-technology communication inside a cell, an ensemble, and inside a community which is characterized by devices using heterogeneous network and service technologies combined by a General Purpose Access Point (GPAP). In our communication scenario the GPAP combines Bluetooth and Ethernet cells using service proxying between Bluetooth SDP and IP-based Web Services. By using multiple GPAPs, remote ensembles can be linked to a pervasive community to allow a service-based communication between members of different ensembles. We used an application for chat and file-transfer to demonstrate our current middleware and look forward to achieve a cross-technology video and audio communication in the future. The support of real-time requirements, roaming and handoff for mobile devices using heterogeneous network and services technologies and our middleware to bridge between these technologies guides our ongoing research.

\section{ACKNOWLEDGMENT}

This research is supported by the German National Science Foundation (DFG, GRK1424).

\section{REFERENCES}

[1] K. Liu, J. Li, P. Huang, and A. Fukuda. Adaptive Acquisition Multiple Access Protocol in Wireless Multihop Mobile Ad Hoc Networks. In Proceedings of the 55th Vehicular Technology Conference (VTC). 2002.

[2] J. LI, Z. Haas, M. Sheng, and Y. Chen. Performance Evaluation of Modified IEEE 802.11 MAC for Multi-Channel Multi-Hop Ad Hoc Networks. 2003.

[3] C. Cordeiro, S. Abhyankar, R. Toshiwal, and D. Agrawal. BlueStar: Enabling Efficient Integration between Bluetooth WPANs and IEEE 802.11 WLANs. ACM/Kluwer Mobile Networks and Applications (MONET) Journal. 2004.

[4] Yujin Lim, Jsung Kim, Sang Lyul Min, and Joong Soo Ma. Performance Evaluation of the Bluetooth-based Public Internet Access Point. In Proceedings of the 15th International Conference on Information Networking (ICOIN). 2001.

[5] E. Dressler, R. Zender, U. Lucke, and D. Tavangarian. A new Architecture for Heterogeneous Context Based Routing. In Appears in Proceedings of the 13th International CSI Computer Conference (CSICC). Kish Island. Iran. March. 2008.

[6] R. Zender, E. Dressler, U. Lucke, and D. Tavangarian. Meta-Service Organization for a Pervasive University. In Proceedings of PerEL 2008. Workshop at 7th IEEE International Conference on Pervasive Computing and Communications (PerCom). March. 2008.

[7] David Sean Mackie. Extending the Reach of Personal Area Networks by Transporting Bluetooth Communications Over IP Networks. http://eprints.ru.ac.za/861/01/mackie-msc-tr07-23.pdf. 2006.

[8] Bluetooth Special Interest Group (SIG). Bluetooth specification. www.bluetooth.com

[9] Park W. et al. Specification of the Bluetooth System. http://bluetooth.com/NR/rdonlyres/F8E8276A-3898-4EC6-B7DAE5535258B056/6545/Core_V21_EDR.zip. July. 2007

[10] BlueZ project. BlueZ. www.bluez.org.

[11] avetana $\mathrm{GmbH}$. avetana Bluetooth JSR 82 implementation. www.avetana-gmbh.de/avetana-gmbh/produkte/jsr82.eng.xml.

[12] The OSGi Alliance. OSGi Service Platform. IOS Press. 2003.

[13] J. Newmarch. Foundations of Jini 2 Programming. Apress, New York. 2006.

[14] Microsoft Corporation. Understanding UPnP: A White Paper. 2000.

[15] Apple Inc. Bonjour Overview. 2006.

[16] S. Weerawarana, F. Curbera, and F. Leymann. Web Services Platform Architecture: Soap, WSDL, WS-Policy, WSAddressing, WS-Bpel, WS Reliable Messaging and More. Prentice Hall International. 2005

[17] Linden Lab. Second Life: Official site of the $3 D$ virtual world. http://secondlife.com. 2008. 